\title{
Genomic profile in gestational and non-gestational choriocarcinomas
}

\author{
Julia Bette Homem de Mello a, Priscila Daniele Ramos Cirilo ${ }^{b}$, Odair Carlito Michelin ${ }^{\text {c }}$, \\ Maria Aparecida Custódio Domingues ${ }^{\mathrm{d}}$, Marilza Vieira Cunha Rudge ${ }^{\mathrm{e}}$, \\ Silvia Regina Rogatto ${ }^{\mathrm{f}, \mathrm{g}, *, 1}$, Izildinha Maestá e, 1 \\ a International Research Center - AC Camargo Cancer Center, São Paulo, SP, Brazil \\ ${ }^{\mathrm{b}}$ Instituto do Câncer do Estado de São Paulo, Faculdade de Medicina, Universidade de São Paulo, SP, Brazil \\ ${ }^{\mathrm{c}}$ Dept de Oncologia Clínica, Faculdade de Medicina, UNESP - Botucatu, SP, Brazil \\ d Dept de Patologia, Faculdade de Medicina, UNESP - Botucatu, SP, Brazil \\ e Dept de Ginecologia e Obstetrícia, Faculdade de Medicina, UNESP - Botucatu, SP, Brazil \\ ${ }^{\mathrm{f}}$ Dept de Urologia, Faculdade de Medicina, UNESP -Botucatu, SP, Brazil \\ ${ }^{g}$ Department of Clinical Genetics, Vejle Sygehus and Institute of Regional Health, University of Southern Denmark, Vejle, Denmark
}

\section{A R T I C L E I N F O}

\section{Article history:}

Received 23 August 2016

Received in revised form

1 December 2016

Accepted 5 December 2016

\section{Keywords:}

Gestational choriocarcinoma

Non-gestational choriocarcinoma

Microsatellites

Array comparative genomic hybridization

Copy number alteration

\begin{abstract}
A B S T R A C T
Introduction: Gestational (GC) (derived from the placenta) and non-gestational (NGC) choriocarcinomas are trophoblastic diseases originated from abnormal proliferation of trophoblastic cells. These rare tumors share similar morphology and pathological features and differ on chemotherapy response, genetic origin and prognosis. In this study, the genomic profile of choriocarcinomas was performed according to their origin (GC or NGC) aiming to better understand these poorly characterized diseases.

Methods: Thirteen patients were included in this study; 10 presented previous history of hydatidiform mole and six developed metastasis. Twelve polymorphic microsatellite markers (D15S659, APOC2, D5S816, BAT25, D3S1614, D3S1311, D1S1656, APC-D5S346, D3S1601, D18S70, D8S1110 and D11S1999) were investigated to distinguish GC from NGC. All choriocarcinomas were evaluated by copy number alterations using array $\mathrm{CGH}$.

Results: Eight cases were classified as GC and five as NGC. Although potentially polymorphic, NGC exhibited significant gain of 21p11. Rare copy number alterations (CNA) were detected as a frequent event in GC including gains of 1p36.33-p36.32 (3 cases), 17q25.3 (4 cases), and losses of 9q33.1 (5 cases), $17 q 21.3$ (3 cases) and 18q22.1 (4 cases) (varying from 724 to 3,053 Kb).

Discussion: Two tumor suppressor genes are candidates to be involved in GC: TRIM32 (9q33.1) and $C D H 19$ (18q22.1). Gains of $C B X 2, C B X 4$ and $C B X 8$ were frequently found in high risk prognostic score in GC. The in silico functional interaction analysis revealed the involvement of PTEN and PI3K-Akt signaling pathways. These data pointed out significant genomic alterations in GC, opening new avenues to better characterize the pathobiology of this disease.
\end{abstract}

() 2016 Elsevier Ltd. All rights reserved.

\section{Introduction}

Trophoblastic diseases originate from abnormal proliferation of trophoblastic cells and encompass different lesions including the choriocarcinoma, which is classified in gestational (GC) and nongestational (NGC) [1]. Gestational choriocarcinoma usually affects

\footnotetext{
* Corresponding author. Dept of Clinical Genetics, Vejle Sygehus, Institute of Regional Health Research (SDU), University of Southern Denmark, Vejle, Denmark. E-mail addresses: silvia.regina.rogatto@rsyd.dk, rogatto@fmb.unesp.br (S.R. Rogatto).

${ }^{1}$ Both authors contributed equally.
}

women at reproductive age and is frequently derived from pregnancies, molar pregnancy, induced and spontaneous abortion, ectopic pregnancy and term or pre-term deliveries [2]. The incidence of GC has been estimated in 1:40.000-50.000 pregnancies, and 1:40 hydatidiform mole cases. The incidence of NGC is unknown, being reported as a very rare tumor $[2,3]$. GC is characterized by the presence of paternal genetic material. These conditions are highly invasive, vascularized and metastatic [4]. Nongestational choriocarcinoma is independent of pregnancies and less responsive to chemotherapy than gestational choriocarcinoma [2].

The pathogenesis of these rare trophoblastic lesions is poorly 
understood. Although differences have been reported in the chemotherapy response, genetic origin and prognosis, GC and NGC exhibit similar morphological pattern, histopathological classification and biochemical markers $[2,5,6]$. In patients with NGC, surgical tumor resection and treatment with multiple chemotherapy agents are indicated. Even with strategies of multidrug therapy, a large number $(\sim 84 \%)$ of patients with NGC died from the disease [6]. Therefore, the difference in therapeutic response and prognosis is clinically relevant and highlight the importance of distinguishing these two entities [2].

Polymorphic microsatellite markers have been used to identify the parental origin of these trophoblastic diseases contributing to therapeutic decision and management of the patients [6-8]. Microsatellites are highly repeated DNA sequences distributed throughout the genome, composed by short sequences of DNA repeated in tandem [9]. Due to the high level of heterozygosis, these markers can differentiate parental alleles with high precision, being considered an efficient molecular strategy to distinguish GC from NGC $[7,8,10]$.

Array Comparative Genomic Hybridization (aCGH) is a screening procedure of genomic imbalances that have the potential to identify recurrent copy-number alterations and its potential as prognostic biomarkers and molecular targets for therapy [11]. To our knowledge, only one study reported genomic alterations in 10 primary GC by aCGH [12]. The authors reported normal genomic profiles in six cases; four of them showed copy number alterations on chromosomes 1,11, 14, 17, 18, 19, 20 and X.

In this study, we used aCGH to characterize and compare the genomic profile of gestational and non-gestational choriocarcinomas, aiming to identify potential molecular markers useful for clinical practice.

\section{Methods}

\subsection{Patients}

Thirteen primary choriocarcinoma samples were enrolled between 1995 and 2009 at the Department of Gynecology and Obstetrics, Botucatu Medical School, UNESP - São Paulo State University, Botucatu, SP, Brazil. The Institutional Human Research Ethics Committee approved this study (Protocol \#3543-2010). All patients were advised of the procedures and provided written consent. Five $\mathrm{mL}$ of peripheral blood samples were collected from 13 patients and her partner/husband (available in 10 cases).

\subsection{Clinical and histopathological characterization}

The clinical data, demographic information, pregnancy history, clinical presentation, management and response to therapy were collected for each patient (Table 1). The prognostic score was based on the International Federation of Gynecology and Obstetrics (FIGO) [13].

Histopathological analysis confirmed epithelial neoplastic biphasic cells composed by mononuclear atypical cells with scant, clear to granular cytoplasm (cytotrophoblast), and multinucleated cells with pleomorphic nucleus (syncytiotrophoblast) (Fig. 1). Extensive area of necrosis and the presence of hemorrhage were frequently observed. Tumor cells were positively stained for AE1/ AE3 (Dako, Glostrup, DK), hCG (Dako, Glostrup, DK), hPL (Dako, Glostrup, DK) and negatively for PLAP (Dako, Glostrup, DK) (detailed information are available in Supplementary Table 1). Cell proliferation rate was evaluated by Ki67 (Dako, Glostrup, DK) immunostaining, varying from 40 to $95 \%$ of nuclei positivity (Table 1).

\subsection{Samples and DNA extraction}

Histological slides stained with hematoxylin-eosin containing the tumor samples were used as reference to guide the tumor microdissection. Non-tumor cells, necrotic cells and hemorrhagic areas were excluded. The tumor tissues were obtained from 10 to 15 unstained slides $(6-10 \mu)$ per case. Genomic DNA from six fresh frozen tumor samples and seven formalin fixed paraffin embedded (FFPE) tumor samples were isolated using the DNeasy Blood \& Tissue Kit (Qiagen, Valencia, USA), according to the manufacturer's instructions. Genomic DNA was isolated from peripheral blood leukocytes using a standard phenol-chloroform (Invitrogen, Life Technologies, CA, USA) extraction and ethanol precipitation procedure.

\subsection{Microsatellite analysis}

A panel of 12 highly polymorphic microsatellite markers (D1S1656, D3S1311, D3S1601, D3S1614, D5S346, D5S816, D8S1110, D11S1999, D15S659, D18S70, APOC2 and BAT25) was selected to evaluate the parental origin of the cases. Supplemental Methods summarizes the PCR conditions.

The fluorescent PCR products were analyzed using ABI 3130XL Genetic Analyzer (Applied Biosystems, Foster City, USA). Each fluorescent peak was quantified by its size (in base pairs), peak height, and peak area as previously reported [14]. The results were analyzed by GeneMapper 4.1 software (Applied Biosystems, Foster City, USA).

\subsection{Array comparative genomic hybridization and data analysis}

Genomic DNA from 13 choriocarcinomas and a male commercial genomic DNA (Promega, Madison, USA) were hybridized on Agilent Human $4 \times 44$ K CGH Microarray (Agilent Technologies, Santa Clara, USA) according to the manufacturer's instructions. Supplemental Methods describes in details the data extraction and the statistical analysis. The genomic alterations obtained in our study were compared with the Database of Genomic Variants (DGV, http://dgv. tcag.ca/dgv/app/home, updated in July 2015). For this comparison, the same type of alteration (gains or losses), same size or overlapping for $>80 \%$ were considered. Rare copy number alterations (observed in less than 10 individuals from the DGV database) detected in at least $50 \%$ of samples were evaluated in more details. The CNAs were also compared with those described in normal placenta [15]. Sexual chromosomes were excluded from the analysis. Chi Square, Mann-Whitney and Fisher exact test $P$ value $\leq 0.05$, (SPSS 18.0, Chicago, USA) was applied to compare the clinical data and genomic alterations.

\subsection{In silico analysis}

The molecular processes, functions and networks were evaluated by the analyses of genes mapped in regions affected by genomic imbalances in gestational choriocarcinoma samples using the Ingenuity Pathways Analysis (IPA) (Ingenuity Systems, http:// www.ingenuity.com) and KOBAS software (v. 2.0; http://kobas. cbi.pku.edu.cn/home.do). Protein-protein interaction (PPI) networks were annotated, visualized and analyzed using NAViGaTOR v2.03 (http://ophid.utoronto.ca/navigator/).

\section{Results}

\subsection{Clinical data}

Ten of 13 presented the tumor located in the uterus (10 cases) 
Table 1

Clinical and histopathological features, stage and prognostic score of 13 choriocarcinomas included in this study.

\begin{tabular}{|c|c|c|c|c|c|c|c|c|c|c|c|c|c|}
\hline Patient & $\mathrm{T} 1$ & $\mathrm{~T} 2$ & T3 & $\mathrm{T} 4$ & $\mathrm{~T} 5$ & T6 & $\mathrm{T} 7$ & $\mathrm{~T} 8$ & T9 & T10 & T11 & $\mathrm{T} 12$ & T13 \\
\hline Age (years) & 28 & 25 & 24 & 36 & 16 & 36 & 26 & 32 & 32 & 23 & 42 & 45 & 26 \\
\hline Tumor Classification & GC & GC & GC & GC & GC & GC & GC & GC & NGC & NGC & NGC & NGC & NGC \\
\hline Tumor Location & Lung & Uterus & Uterus & Uterus & Uterus & Uterus & $\begin{array}{l}\text { Uterine } \\
\text { cervix }\end{array}$ & Uterus & Uterus & Uterus & Uterus & Uterus & Uterus \\
\hline Metastasis & Liver & - & Vagina & - & Lung & Lung & $\begin{array}{l}\text { Uterine } \\
\text { cervix }\end{array}$ & - & - & $\begin{array}{l}\text { Lung/ } \\
\text { Vagina }\end{array}$ & - & - & - \\
\hline Number of Pregnancies & 2 & 3 & 1 & 4 & 2 & 2 & 3 & 3 & 4 & 3 & 7 & 4 & 3 \\
\hline Number of Live-births & 1 & 1 & 0 & 3 & 0 & 0 & 2 & 2 & 3 & 3 & 3 & 3 & 1 \\
\hline History of pregnancy & HM & HM & $\mathrm{HM}$ & Abortion & HM & HM & Abortion & $\mathrm{HM}$ & HM & $\begin{array}{l}\text { Term } \\
\text { pregnancy }\end{array}$ & HM & HM & HM \\
\hline $\begin{array}{l}\text { Period between last } \\
\text { pregnancy and beginning } \\
\text { of treatment (months) }\end{array}$ & 56 & 12 & 6 & 25 & 24 & 18 & 18 & 4 & 24 & 14 & 6 & 8 & 16 \\
\hline $\begin{array}{l}\text { Pretreatment serum hCG } \\
\quad(\mathrm{mUI} / \mathrm{mL})\end{array}$ & 52,582 & 79,530 & 10,349 & 182,939 & 302,409 & 3103 & 15,406 & 82,000 & 35,849 & 814,108 & 25,051 & 27,303 & 29,227 \\
\hline Prognostic score & 13 & 9 & 4 & 11 & 10 & 3 & 7 & 3 & 3 & 8 & 3 & 5 & 5 \\
\hline Prognostic score risk & High & High & Low & High & High & Low & High & Low & Low & High & Low & Low & Low \\
\hline Chemotherapy protocol & $\begin{array}{l}\text { EMA/CO; EP/ } \\
\text { EMA; Ifosfamide } \\
\text { paclitaxel }\end{array}$ & $\begin{array}{l}\text { EMA/ } \\
\text { CO; EP/ } \\
\text { EMA }\end{array}$ & MTX/FA & EP/EMA & EP/EMA & MTX/FA & EP/EMA & Act-D & $\begin{array}{l}\text { MTX/ } \\
\text { FA; Act- } \\
\text { D }\end{array}$ & EP/EMA & MTX/FA & MTX/FA & $\begin{array}{l}\text { MTX/FA; } \\
\text { Act-D }\end{array}$ \\
\hline Resistance to chemotherapy & Yes & Yes & No & No & No & No & No & No & Yes & No & No & No & Yes \\
\hline Follow up (months) & 36 & 24 & 27 & 24 & 45 & 32 & 50 & 60 & 60 & 66 & 65 & 12 & 60 \\
\hline Death & Yes & No & No & No & No & No & No & No & No & No & No & No & No \\
\hline Cellular composition & Biphasic & Biphasic & Biphasic & Biphasic & Biphasic & Biphasic & Biphasic & Biphasic & Biphasic & Biphasic & Biphasic & Biphasic & Biphasic \\
\hline Cellular necrosis & Ample & Ample & NA & Ample & Ample & Ample & Ample & Ample & Ample & NA & Absent & Ample & Ample \\
\hline Hemorrhage & Severe & Severe & NA & Absent & Severe & NA & Severe & Severe & Severe & NA & NA & NA & Absent \\
\hline Invasion of the blood vessels & + & - & NA & - & + & - & - & + & + & NA & + & + & + \\
\hline Myometrium invasion depth & - & $<1 / 3$ & NA & $<1 / 2$ & $\begin{array}{l}\text { Uterine } \\
\text { serosa } \\
\text { invasion }\end{array}$ & $<1 / 3$ & - & $\begin{array}{l}\text { Uterine } \\
\text { serosa } \\
\text { invasion }\end{array}$ & $<1 / 2$ & NA & $<1 / 3$ & $<1 / 3$ & $\begin{array}{l}\text { Uterine } \\
\text { serosa } \\
\text { invasion }\end{array}$ \\
\hline AE1/AE3 & + & + & + & + & + & + & + & NA & + & NA & + & - & NA \\
\hline hCG & +++ & +++ & ++ & +++ & +++ & +++ & ++ & NA & +++ & NA & +++ & +++ & NA \\
\hline hPL & + & + & + & + & + & + & + & NA & - & NA & + & + & NA \\
\hline Ki-67 & $>95 \%$ & $80 \%$ & NA & $>70 \%$ & $80 \%$ & - & $40 \%$ & NA & $>50 \%$ & NA & $40 \%$ & $40 \%$ & NA \\
\hline PLAP & - & + (focal) & $\begin{array}{l}+ \\
\text { (focal) }\end{array}$ & - & - & - & - & NA & - & NA & - & - & NA \\
\hline
\end{tabular}

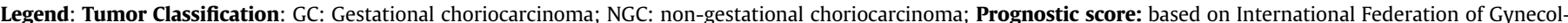

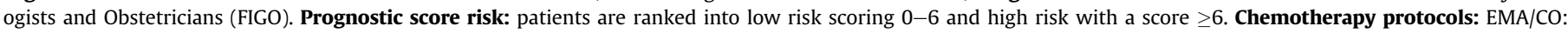

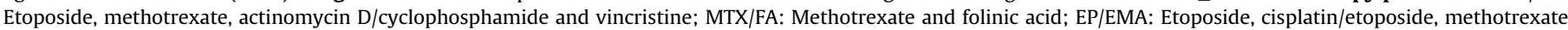

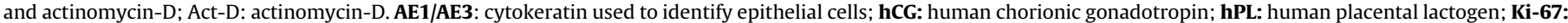

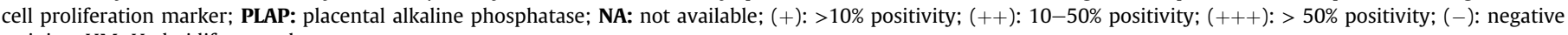
staining. HM: Hydatidiform mole.

and six cases developed metastasis, especially at lung and vagina ( 5 of $8 \mathrm{GC}$ and 1 of $5 \mathrm{NGC}$ ). Hydatidiform mole was found in 10 patients ( 6 of 8 GC and 4 of 5 NGC). Four patients presented chemotherapy resistance to the first line of chemotherapy (2 GC and 2 NGC). Second line chemotherapy protocol using EP/EMA were applied to patients $\mathrm{T} 1$ and T2, and Act-D for patients T9 and T3. Only the patient $\mathrm{T} 1$ remained resistant to treatment, which was applied a third line of treatment. However, she died with multiple hepatic lesions and liver failure 3 years after being diagnosed with choriocarcinoma. The detailed clinical analysis of the patient T1 was previously reported [16]. High prognostic score (according to FIGO) was found in six cases (5 GC and 1 NGC). Detailed clinical data of the patients are shown in Table 1 . Using these clinical data, no significant differences were observed between GC and NGC (Supplementary Table 2).

\subsection{Microsatellite markers characterization}

All 13 cases had informative results from the analysis of 12 microsatellite markers (Table 2). Eight cases presented bi-parental alleles (GC) and five cases had exclusively maternal alleles (NGC) (Fig. 2A and B).

\subsection{Genomic alterations}

A total of 200 CNA affecting all chromosomes was observed in GC and NGC samples. Eight GC samples exhibited 157 CNA, being 66 gains, 1 high copy-number gain, and 90 losses. Among the five NGC samples, 43 CNA were detected including 22 gains and 21 losses. An overall overview of genomic alterations in GC and NGC revealed distinct profiles with higher number imbalances in GC (Fig. 2C). Significant alterations classified as rare were selected for more detailed analysis (Table 3).

Gestational choriocarcinomas presented nine significant rare CNAs including: gains of $1 \mathrm{p} 36.33-\mathrm{p} 36.32,8 \mathrm{q} 24.3,10 \mathrm{q} 24.32$, 17q25.3, 18p11.1-q11.1, 21p11.1; and losses of 9q33.1, 17q21.33, 18q22.1. Gain of 21p11.1 was the only significant rare CNA observed in two NGC (Table 3). The comparison with the data recently published in normal placenta [15] revealed that gains of 10q24.32, 17q25.3, 18p11.1-q11.1 and 21p11.1 and losses of 9q33.1 and $17 q 21.33$ were exclusively present in GC cases. The loss of 18q22.1 found on our cases overlapped a small region $(1,3 \mathrm{~Kb})$ in normal placenta (without known genes mapped in this region).

\subsection{In silico functional analysis}

The list of 187 genes affected by CNA detected in GC samples was 

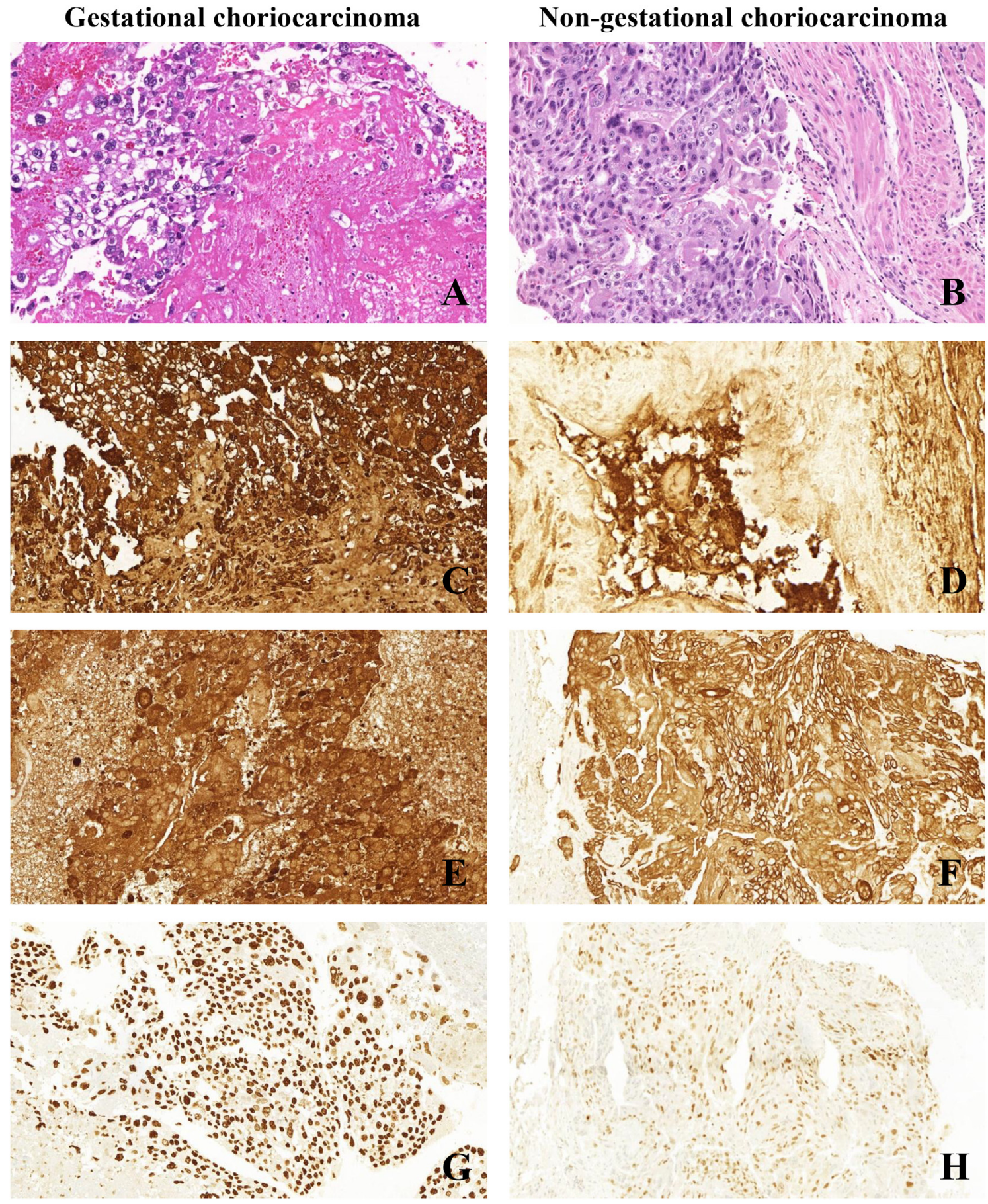

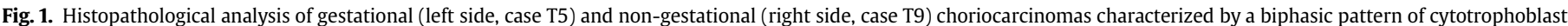

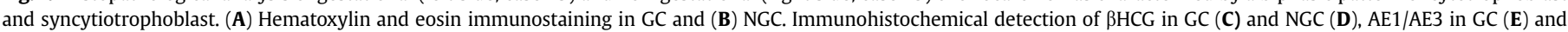
NGC (F), and Ki67 immunoexpression in GC $(\mathbf{G})$ and NGC $(\mathbf{H})$.

evaluated with the IPA and KOBAS software. The NF-KB activation (NFKB2, TNFRSF14, ITGA3, PRKCZ), Tec kinase (TLR4, NFKB2, ITGA3, GNB1, PRKCZ) and PTEN signaling pathways (ITGA3, SHARPIN, NFKB2 and $P R K C Z$ ), frequently reported in several tumor types, were observed among the deregulated canonical pathways (Supplementary Table 3). The PTEN pathway was confirmed as altered in both analyses. Biological functions as organism development, cell-to-cell signaling and interaction, cellular assembly and organization were observed among the deregulated networks (Supplementary Table 4, Fig. 3A).
Protein-protein interaction (PPI) network analysis was performed using 29 genes mapped on 9q33.1, 17q25.3 and 18q22.1 affected by CNA detected in at least 50\% of samples. EIF4A3 protein was highly inter-connected with other proteins, mostly associated with transcription regulation. Proteins codified by $C D H 19$ and $C D H 7$ co-interacting and are also correlated with several proteins involved in cellular organization. CBX protein family co-interaction was connected with proteins involved in several biological functions, as genome maintenance and transcription regulation (Fig. 3B). 
Table 2

Data obtained from the analysis of 12 microsatellite markers used to categorize the samples in gestational (GC) and non-gestational choriocarcinomas (NGC).

\begin{tabular}{|c|c|c|c|c|c|c|c|c|c|c|c|c|}
\hline Case & D15S659 & APOC2 & D5S816 & BAT25 & D3S1614 & D3S1311 & D1S1656 & APC-D5S346 & D3S1601 & D18S70 & D8S1110 & D11S1999 \\
\hline $\mathrm{T} 1$ & GC & $\mathrm{NI}$ & NI & GC & $\mathrm{NI}$ & GC & GC & $\mathrm{NI}$ & GC & $\mathrm{NI}$ & GC & GC \\
\hline $\mathrm{T} 2$ & GC & GC & $\mathrm{NI}$ & GC & GC & GC & $\mathrm{NI}$ & GC & GC & GC & GC & $\mathrm{NI}$ \\
\hline T3 & GC & GC & GC & GC & NI & NI & $\mathrm{NI}$ & NI & $\mathrm{NI}$ & GC & GC & GC \\
\hline $\mathrm{T} 4$ & GC & GC & NI & $\mathrm{NI}$ & $\mathrm{NI}$ & $\mathrm{NI}$ & $\mathrm{NI}$ & GC & GC & NI & $\mathrm{NI}$ & GC \\
\hline T5 & NI & NI & NI & NI & $\mathrm{NI}$ & GC & GC & GC & $\mathrm{NI}$ & NI & NI & NI \\
\hline T6 & GC & GC & GC & GC & $\mathrm{NI}$ & GC & GC & GC & GC & GC & GC & $\mathrm{NI}$ \\
\hline $\mathrm{T} 7$ & GC & GC & $\mathrm{NI}$ & GC & GC & NI & $\mathrm{NI}$ & $\mathrm{NI}$ & $\mathrm{NI}$ & GC & GC & GC \\
\hline T8 & $\mathrm{NI}$ & NI & GC & $\mathrm{NI}$ & $\mathrm{NI}$ & $\mathrm{NI}$ & $\mathrm{NI}$ & GC & $\mathrm{NI}$ & $\mathrm{NI}$ & $\mathrm{NI}$ & GC \\
\hline T9 & NGC & $\mathrm{NI}$ & $\mathrm{NI}$ & $\mathrm{NI}$ & $\mathrm{NI}$ & $\mathrm{NI}$ & NGC & $\mathrm{NI}$ & NGC & NGC & $\mathrm{NI}$ & NI \\
\hline T10 & NGC & NGC & $\mathrm{NI}$ & NI & NGC & NGC & $\mathrm{NI}$ & $\mathrm{NI}$ & NGC & NGC & NI & NI \\
\hline T11 & NI & NGC & $\mathrm{NI}$ & $\mathrm{NI}$ & NGC & $\mathrm{NI}$ & NGC & NGC & $\mathrm{NI}$ & NGC & NGC & $\mathrm{NI}$ \\
\hline T12 & $\mathrm{NI}$ & NGC & NGC & NGC & NI & $\mathrm{NI}$ & NGC & NGC & $\mathrm{NI}$ & $\mathrm{NI}$ & $\mathrm{NI}$ & NGC \\
\hline T13 & NGC & NGC & NI & NI & $\mathrm{NI}$ & $\mathrm{NI}$ & NGC & NGC & NGC & NGC & $\mathrm{NI}$ & NI \\
\hline
\end{tabular}

*NI = non-informative; GC = gestational choriocarcinoma; NGC = non-gestational choriocarcinoma.

\section{Discussion}

The distinction between gestational and non-gestational choriocarcinoma has been proved as important for the establishing of an appropriate treatment, to predict the response to therapy as well as for prognosis [6,17]. Over the past decade, microsatellite markers have been used as an efficient tool to differentiate GC and NGC $[7,8]$. In our study, a panel of 12 polymorphic microsatellite markers was used to categorize eight GC and five NGC cases. Five of eight GC developed metastasis (mostly in lung and vagina) compared with one NGC that developed metastasis in lung and vagina. Similarly to our data, the literature shows that approximately $50 \%$ of patients develop metastatic choriocarcinomas $[4,18]$. Furthermore, in both groups, the majority of cases presented previous hydatidiform mole ( 6 of 8 GC and 4 of 5 NGC). Strohl et al. [19] reported that approximately $90 \%$ of patients with low-risk of gestational trophoblastic neoplasia and $40 \%$ of cases with choriocarcinomas are preceded by hydatidiform mole. However, to our knowledge, no epidemiological studies have been performed to evaluate the incidence of previous hydatidiform mole in patients with NGC.

Large-scale genomic analysis has revealed CNA that potentially contribute to the development or progression of several tumor types. Recently, Kasak et al. [15] described an extensive number of somatic CNVs in normal placenta showed (mostly duplications) suggesting that this event is critical for normal gestation. Interestingly, gain of 1 p36.33-p36.32 described by the authors was found in our GC. Although with no genes described, gain of 8q24.3 partially $(100 \mathrm{~Kb})$ overlapped the same region reported in normal placenta. Overall, several alterations herein described in GC have potential to be associated with the development and progression of this malignant trophoblastic disease.

To our knowledge, only one study reported 10 primary GC evaluated by aCGH (244 K, Agilent Technologies) [12]. The authors described four samples with 1-11 CNA, while six cases had no alterations. Alteration of 9q33.1 (5 cases) and 18q22.1 (4 cases) were found in common in accordance to our data.

Three regions significantly altered in our GC cases and absent in normal placental tissues were selected for detailed discussion: loss of 9q33.1 (5 cases), 18q22.1 (4 cases) and gains of 17q25.3 (4 cases).

Loss of 9q33.1 encompasses nine genes including TRIM32, described in literature as a pro-apoptotic factor and tumor suppressor gene [20]. The Trim32 ubiquitinates X-linked inhibitor of apoptosis (XIAP) promotes proteosomal degradation [20]. XIAP is a well-known cancer therapeutic target and down-regulation of this protein may increase the sensitivity of cell chemoresistance to cisplatin-induced apoptosis [21]. We detected genomic loss involving TRIM32 in five GCs, while two of these patients that were treated with a combination of drugs, including cisplatin, presented resistance to chemotherapy. These findings suggest that TRIM32 loss is a putative marker associated with the mechanism of treatment response in GC. The loss in 9q33.1 also includes PAPPA, which is normally down-regulated in response to the early embryonic signal, human chorionic gonadotropin (hCG) [22]. The PAPPA loss could be indirectly related with hCG increased levels in a number of patients with gestational choriocarcinomas.

A significant loss of 18q22.1 (3.1 Mb) harboring $C D H 7$ and $C D H 19$ genes was detected in four GC samples. Loss involving 18q21.3-q23 was previously described in one primary choriocarcinoma [12]. Kasak et al. [15] found duplication of $C D H 19$ in normal placenta giving additional support of the involvement of this gene in choriocarcinomas. Down-regulation of $\mathrm{CDH} 19$ was associated with advanced head and neck squamous cell carcinomas and colorectal tumors [23,24]. Taken together, our findings suggest that $C D H 7$ and CDH19 are potential contributors to the gestational choriocarcinoma development.

Fourteen genes mapped on 17q25.3 were involved in gains (cases T1, T2, T4 and T8), including CBX2, CBX4 and CBX8. Proteinprotein interaction (PPI) networks prediction demonstrated that these proteins co-interact and are important components of Polycomb repressive complexes. The polycomb group (PcG) proteins are known to transcriptionally silence crucial tumor suppressor genes in a variety of cancers through histone modification [25]. Interestingly, three of four cases with 17q25.3 gains were classified as having high risk prognostic score (>6) (according to FIGO 2000) giving support for the involvement of this alteration in GC.

In silico functional interaction analysis demonstrated that PTEN signaling pathway may display an important role in GC. We detected genomic gains involving three genes (SHARPIN, NFKB2 and $P R K C Z)$ responsible for the PTEN pathway negative regulation [26-28]. In addition, four GC patients showing alterations in genes associated with the PTEN pathway were classified as high risk prognostic score $(>6)$. These data suggested that PTEN signaling pathway might have an important role in gestational choriocarcinomas.

The in silico analysis also revealed the involvement of the PI3KAkt signaling pathway (ITGA3, COL1A1, CHAD, TLR4, GNB1 and $P R K C Z$ ) in GC. This pathway involves proteins negatively regulated by PTEN through AKT inhibition and acts as regulator of cell growth, proliferation, migration and adhesion $[29,30]$. Recently, the anticancer effects of apigenin were investigated in choriocarcinoma cell lines (JAR and JEG3) [31]. According to the authors, apigenin promotes activation of apoptosis and inhibition of cell proliferation and migration, acting via inactivation of PI3K/AKT and activation of 

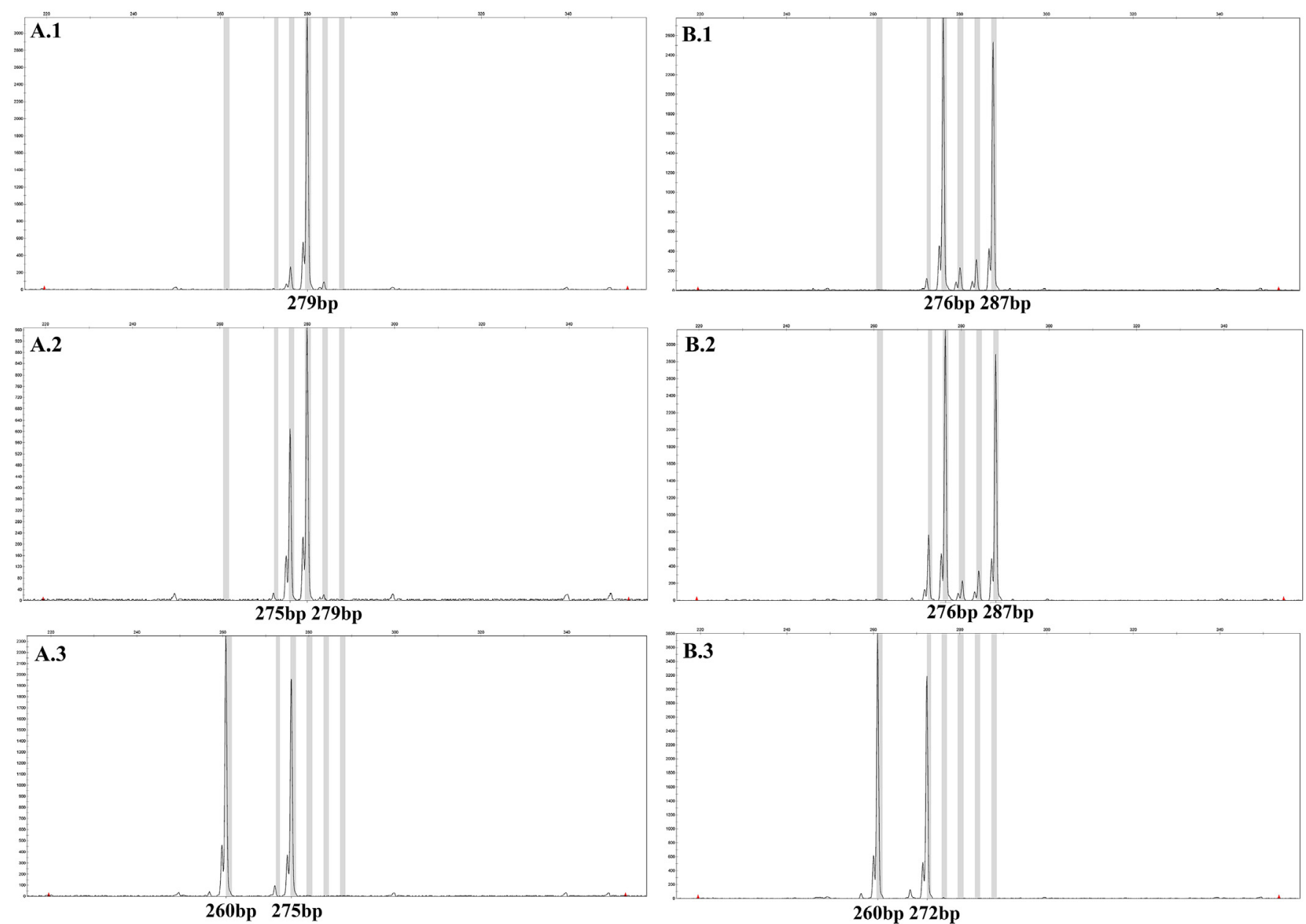

C

Chromosomes

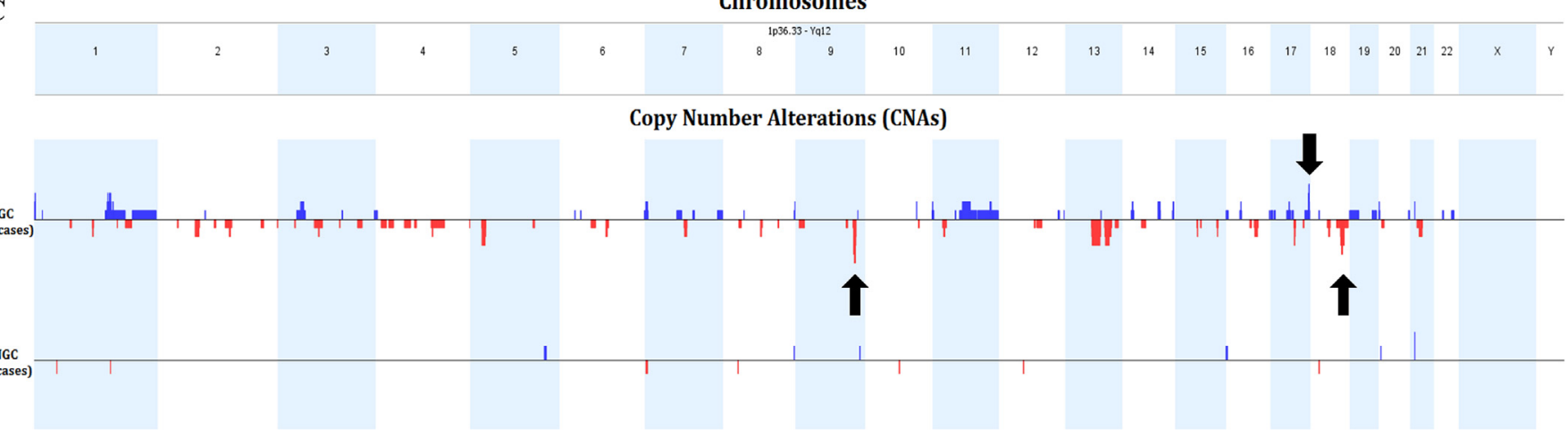

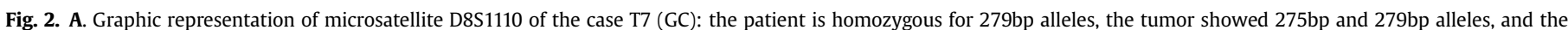

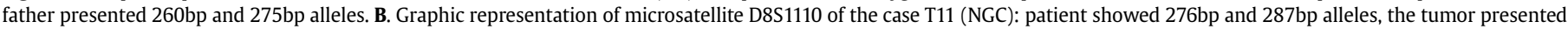

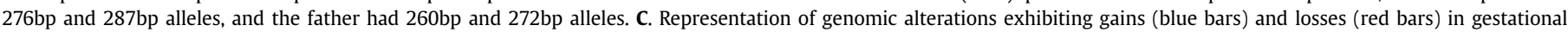

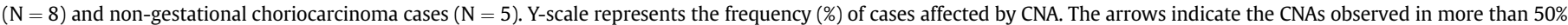
of GC cases.

ERK1/2 signaling pathways [31]. These data highlighted the importance of PI3K-Akt signaling pathway on GC, which brings possibilities to identify better therapeutic strategies for these patients.

Differently from GC, non-gestational choriocarcinomas have been associated with resistance to chemotherapy and reduced survival [32,33]. In our cohort of five NGC cases, none of them died from the disease. Two NGC patients had resistance to the first line of chemotherapy protocol (methotrexate and folinic acid) but had positive response to the second line of treatment using actinomycin-D (follow up of 60 months).

Non-gestational choriocarcinomas exhibited a homogeneous genetic profile, showing few CNAs per sample. The only statistically significant CNA detected in NGC cases was gain of 21p11.1, which was also observed in GC samples. The size (104.6 Kbp) and the absence of genes known in this region suggested that this alteration is a copy number polymorphism. A different mechanism of genetic or epigenetic dysregulation probably is associated with the 
Table 3

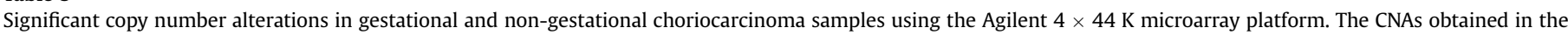
analysis were compared with the Database of Genomic Variants (DGV, http://dgv.tcag.ca/dgv/app/home, updated in July 2015).

\begin{tabular}{|c|c|c|c|c|c|c|c|}
\hline Region & Event & $\begin{array}{l}\text { Region } \\
\text { length } \\
\text { (Kb) }\end{array}$ & $\begin{array}{l}\text { Start - end } \\
(\mathrm{Kb})\end{array}$ & Genes & $\begin{array}{l}\mathrm{P} \\
\text { value }\end{array}$ & $\begin{array}{l}\text { Samples } \\
(\mathrm{N}=8)\end{array}$ & DGV* \\
\hline $\begin{array}{r}1 \mathrm{p} 36.33- \\
\mathrm{p} 36.32\end{array}$ & Gain & 896.2 & $\begin{array}{l}1,793,546 \\
-2,689,845\end{array}$ & $\begin{array}{l}\text { GNB1, CALML6, TMEM52, C1orf222, KIAA1751, CFAP74, AK054708, GABRD, PRKCZ, AX747530, } \\
\text { BC018779, C1orf86, SKI, LOC100129534, MORN1, RER1, PEX10, PLCH2, PANK4, HES5, LOC115110, } \\
\text { LOC100133445, TNFRSF14, LOC100996583, FAM213B, MMEL1, TTC34 }\end{array}$ & $>0.001$ & $\mathrm{~T} 1, \mathrm{~T} 4, \mathrm{~T} 5$ & 0 \\
\hline $8 q 24.3$ & Gain & 1063.8 & $\begin{array}{l}144,964,149 \\
-146,027,963\end{array}$ & $\begin{array}{l}\text { PLEC, PARP10, OPLAH, GPAA1, CYC1, PLEC, PARP10, GRINA, SPATC1, OPLAH, EXOSC4, GPAA1, CYC1, } \\
\text { SHARPIN, MAF1, KIAA1875, FAM203A, MROH1, SCXB, SCX, BOP1, HGH1, HEATR7A, BOP1, HSF1, DGAT1, } \\
\text { SCRT1, TMEM249, FBXL6, SLC52A2, ADCK5, CPSF1, SLC39A4, VPS28, TONSL, AK298596, LOC100287098, } \\
\text { CYHR1, KIFC2, FOXH1, PPP1R16A, AK094577, GPT, MFSD3, RECQL4, DQ579335, LRRC14, LRRC24, } \\
\text { C8orf82, ARHGAP39, DQ588968, ZNF251, ZNF34, RPL8,, DL491750, ZNF517, miR-661, miR-6846, miR- } \\
\text { 6847, miR-7112, miR-6848, miR-939, miR-1234, miR-6849, miR-6893 }\end{array}$ & 0.027 & $\mathrm{~T} 1, \mathrm{~T} 2$ & 1 \\
\hline $10 q 24.32$ & Gain & 669.2 & $\begin{array}{l}103,723,394 \\
-104,392,647\end{array}$ & $\begin{array}{l}\text { C10orf76, HPS6, LDB1, PPRC1, NOLC1, ELOVL3, PITX3, BC068543, GBF1, NFKB2, PSD, FBXL15, CUEDC2, } \\
\text { C10orf95, RPARP-AS1, LOC100505761, AX746750, TMEM180, ACTR1A, SUFU, miR-146b }\end{array}$ & 0.015 & T1, T2 & 0 \\
\hline 17q25.3 & Gain & 724.2 & $\begin{array}{l}77,414,421 \\
-78,138,718\end{array}$ & $\begin{array}{l}\text { RBFOX3, HP09025, ENPP7, CBX2, CBX8, CBX4, LOC101928766, BC044939, LOC101928738, TBC1D16, } \\
\text { CCDC40, GAA, EIF4A3, miR-4739 }\end{array}$ & 0.001 & $\begin{array}{l}\mathrm{T} 1, \mathrm{~T} 2 \\
\mathrm{~T} 4, \mathrm{~T} 8\end{array}$ & 0 \\
\hline $\begin{array}{c}\text { 18p11.1 - } \\
\text { q11.1 }\end{array}$ & Gain & 406.1 & $\begin{array}{l}16,793,851 \\
-17,200,000\end{array}$ & - & 0.015 & T6 & 0 \\
\hline 21p11.1 & Gain & 104.6 & $\begin{array}{l}10,013,263 \\
-10,117,957\end{array}$ & - & 0.015 & T6, T8 & 0 \\
\hline 9q33.1 & Loss & 3053.6 & $\begin{array}{l}118,689,745 \\
-121,743,376\end{array}$ & PAPPA, PAPPA-AS1, ASTN2-AS1, LOC100128505, TRIM32, ASTN2, SNORA70C, LOC101928797, TLR4 & $>0.001$ & $\begin{array}{l}\mathrm{T} 1, \mathrm{~T} 2, \\
\mathrm{~T} 4, \mathrm{~T} 6, \mathrm{~T} 8\end{array}$ & 0 \\
\hline $17 q 21.33$ & Loss & 1508.4 & $\begin{array}{l}47,627,158 \\
-49,135,599\end{array}$ & $\begin{array}{l}\text { LOC100288866, NXPH3, SPOP, SLC35B1, FAM117A, KAT7, TAC4, FLJ45513, DLX4, DLX3, LOC284080, } \\
\text { ITGA3, PDK2, SAMD14, PPP1R9B, SGCA, HILS1, BC126901, BC033456, DD181999, HV555657, COL1A1, } \\
\text { LOC101927230, TMEM92, TMEM92-AS1, XYLT2, MRPL27, EME1, DQ599569, LRRC59, ACSF2, CHAD, } \\
\text { RSAD1, MYCBPAP, EPN3, SPATA20, CACNA1G-AS1, CACNA1G, ABCC3, BC131755, ANKRD40, LUC7L3, } \\
\text { AK090674, LINC00483, WFIKKN2, TOB1, TOB1-AS1, SPAG9, miR-8059 }\end{array}$ & 0.018 & $\mathrm{~T} 1, \mathrm{~T} 4, \mathrm{~T} 8$ & 0 \\
\hline $18 \mathrm{q} 22.1$ & Loss & 3129.1 & $\begin{array}{l}61,660,093 \\
-64,789,199\end{array}$ & LINC00305, LOC400654, LOC284294, CDH7, CDH19, miR-5011 & 0.045 & $\begin{array}{l}\text { T1, T2, } \\
\text { T6, T8 }\end{array}$ & 1 \\
\hline \multicolumn{8}{|c|}{ Non-gestational Choriocarcinoma } \\
\hline Region & Event & $\begin{array}{l}\text { Region } \\
\text { length } \\
(\mathrm{Kb})\end{array}$ & $\begin{array}{l}\text { Start - end } \\
(\mathrm{Kb})\end{array}$ & Genes & $\begin{array}{l}\mathrm{P} \\
\text { value }\end{array}$ & $\begin{array}{l}\text { Samples } \\
(\mathrm{N}=5)\end{array}$ & DGV* \\
\hline 21p11.1 & Gain & 104.6 & $\begin{array}{l}10,013,263 \\
-10,117,957\end{array}$ & - & 0.015 & T10, T13 & 0 \\
\hline
\end{tabular}

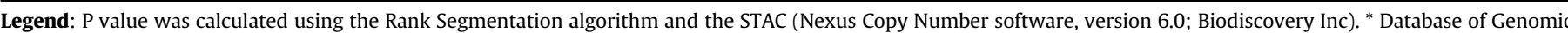

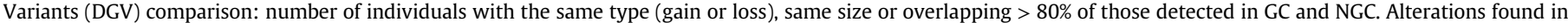
more than 10 individuals of the CNV dataset were exclude from the analysis.

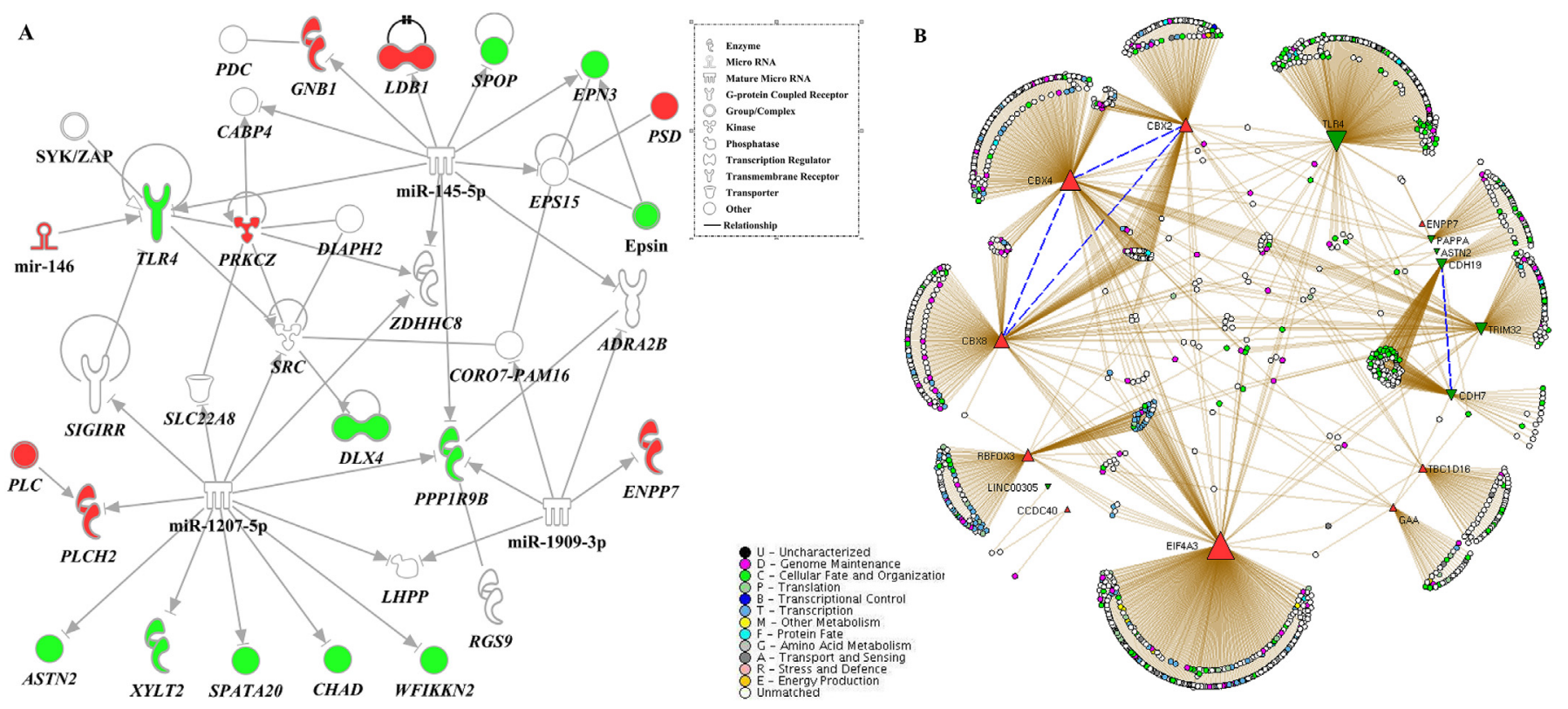

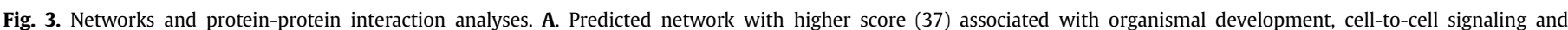

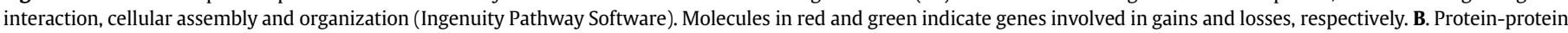

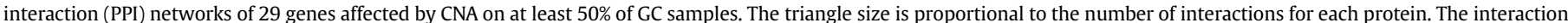

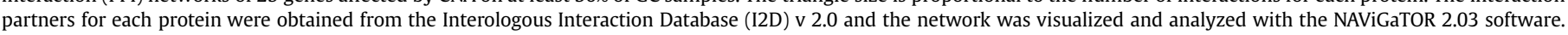
Blue dashed lines represent direct interactions between proteins of interest. 
tumorigenesis of NGC. Therefore, further studies using alternative approaches to better characterize NGS are extremely necessary to better understand and treat these patients.

Since the trophoblastic diseases (GC and NGC) are rare, one limitation of our study is the small number of cases. In addition, the germline genomic profiles were not assessed and the sex chromosomes were excluded of the analysis. However, our results described genomic regions and genes altered in high frequencies with potential involvement in GC samples.

Overall, our data contributes to the understanding of the molecular pathobiology of gestational choriocarcinomas pointing out to the involvement of TRIM32, PAPPA, (both mapped in 9q33.1), $\mathrm{CDH7}$ and $\mathrm{CDH} 19$ (both mapped in 18q22.1) and the Polycomb repressive complexes ( $C B X 2, C B X 4$ and $C B X 8$; mapped in 17q25.3). The PTEN and PI3K-Akt pathways were the most relevant signaling pathways involved in GC. In our cohort of non-gestational choriocarcinoma cases, copy number alterations were not significant and the genetic mechanism associated with these lesions remains to be clarified.

\section{Conflict of interest statement}

The authors declare that there are no conflicts of interest.

\section{Funding}

This study was supported by grants from Fundação de Amparo à Pesquisa do Estado de São Paulo-FAPESP (2010/05926-0); and CNPq (302606/2011-4).

\section{Acknowledgments}

The authors would like to thank the patients that have participated in this study. Our special acknowledgement goes to Rolando Andre Villacis, PhD, Fabio Albuquerque Marques, $\mathrm{PhD}$, and Maisa Pinheiro PhD, for their helpful suggestions during the study.

\section{Appendix A. Supplementary data}

Supplementary data related to this article can be found at http:/ dx.doi.org/10.1016/j.placenta.2016.12.009.

\section{References}

[1] N.R. Desai, S. Gupta, R. Said, P. Desai, Q. Daí, Choriocarcinoma in a 73-year-old woman: a case report and review of the literature, J. Med. Case Rep. 4 (2010) 379.

[2] L. Hoffner, U. Surti, The genetics of gestational trophoblastic disease: a rare complication of pregnancy, Cancer Genet. 205 (3) (2012) 63-77.

[3] O. Wang, C. Guo, L. Zou, Y. Wang, X. Song, Y. Ma, et al., Clinicopathological analysis of non-gestational ovarian choriocarcinoma: report of two cases and review of the literature, Oncol. Lett. 11 (4) (2016) 2599-2604.

[4] M.J. Seckl, N.J. Sebire, R.S. Berkowitz, Gestational trophoblastic disease, Lancet 376 (2010) 717-729.

[5] A.N. Cheung, H.J. Zhang, W.C. Xue, M.K. Siu, Pathogenesis of choriocarcinoma: clinical, genetic and stem cell perspectives, Future Oncol. 5 (2) (2009) 217-231.

[6] E.S. Newlands, The management of recurrent and drug-resistant gestational trophoblastic neoplasia (GTN), Best. Pract. Res. Clin. Obstet. Gynaecol. 17 (6) (2003) 905-923.

[7] R.A. Fisher, P.M. Savage, C. MacDermott, J. Hook, N.J. Sebire, I. Lindsay, et al., The impact of molecular genetic diagnosis on the management of women with hCG-producing malignancies, Gynecol. Oncol. 107 (3) (2007) 413-419.

[8] Y. Wang, Y. Yang, F. Teng, $H$, Zhang, F, Xue, Pure nongestational uterin choriocarcinoma in a postmenopausal Chinese woman confirmed with short tandem repeat analysis, Am. J. Obstet. Gynecol. 211 (1) (2014) e1-3.

[9] M.R. Speicher, Microsatellite instability in human cancer, Oncol. Res. 7 (6)
(1995) 267-275

[10] N. Shahib, D. Martaadisoebrata, H. Kondo, Y. Zhou, N. Shinkai, C. Nishimura, et al., Genetic origin of malignant trophoblastic neoplasms analyzed by sequence tag site polymorphic markers, Gynecol. Oncol. 81 (2001) 247-253.

[11] A.F. Busso-Lopes, F.A. Marchi, H. Kuasne, C. Scapulatempo-Neto, J.C. TrindadeFilho, C.M. de Jesus, et al., Genomic profiling of human penile carcinoma predicts worse prognosis and survival, Cancer Prev. Res. (Phila) 8 (2) (2015) 149-156.

[12] H. Poaty, P. Coullin, J.F. Peko, P. Dessen, A.L. Diatta, A. Valent, et al., Genomewide high-resolution aCGH analysis of gestational choriocarcinomas, PLoS One 7 (1) (2012) e29426.

[13] E.I. Kohorn, The new FIGO 2000 staging and risk factor scoring system for gestational trophoblastic disease: description and critical assessment, Int. J. Gynecol. Cancer 11 (1) (2001) 73-77.

[14] R.A. Canevari, A. Pontes, S.R. Rogatto, Microallelotyping defines novel regions of loss of heterozygosity in uterine leiomyomas, Mol. Carcinog. 42 (2005) $177-182$.

[15] L. Kasak, K. Rull, P. Vaas, P. Teesalu, M. Laan, Extensive load of somatic CNVs in the human placenta, Sci. Rep. 10 (5) (2015) 8342.

[16] I. Maestá, F.V. Leite, O.C. Michelin, S.R. Rogatto, Primary pulmonary choriocarcinoma after human chorionic gonadotropin normalization following hydatidiform mole: a report of two cases, J. Reprod. Med. 55 (7-8) (2010) $311-316$.

[17] J. Zhao, Y. Xiang, X.R. Wan, F.Z. Feng, Q.C. Cui, X.Y. Yang, Molecular genetic analyses of choriocarcinoma, Placenta 30 (9) (2009) 816-820.

[18] G. Mangili, D. Lorusso, J. Brown, J. Pfisterer, L. Massuger, M. Vaughan, et al., Trophoblastic disease review for diagnosis and management: a joint report from the international society for the study of trophoblastic disease, European organisation for the treatment of trophoblastic disease, and the gynecologic cancer interGroup, Int. J. Gynecol. Cancer 24 (2014) S109-S116.

[19] A.E. Strohl, J.R. Lurain, Postmolar choriocarcinoma: an independent risk factor for chemotherapy resistance in low-risk gestational trophoblastic neoplasia, Gynecol. Oncol. 141 (2) (2016) 276-280.

[20] Y.S. Ryu, Y. Lee, K.W. Lee, C.Y. Hwang. J. Maeng J. Kim, et al, TRIM32 protein sensitizes cells to tumor necrosis factor TNF-induced apoptosis via its RING domain-dependent E3 ligase activity against X-linked inhibitor of apoptosis (XIAP), J. Biol. Chem. 286 (29) (2011) 25729-25738.

[21] J. Li, Q. Feng, J.M. Kim, D. Schneiderman, P. Liston, M. Li, et al., Human ovarian cancer and cisplatin resistance: possible role of inhibitor of apoptosis proteins, Endocrinology 142 (1) (2001) 370-380.

[22] J.R. Sherwin, J.M. Hastings, K.S. Jackson, P.A. Mavrogianis, A.M. Sharkey, A.T. Fazleabas, The endometrial response to chorionic gonadotropin is blunted in a baboon model of endometriosis, Endocrinology 151 (10) (2010) 4982-4993.

[23] H. Blons, O. Laccourreye, A.M. Houllier, F. Carnot, D. Brasnu, P. Beaune, et al. Delineation and candidate gene mutation screening of the $18 \mathrm{q} 22$ minimal region of deletion in head and neck squamous cell carcinoma, Oncogene 21 (2002) 5016-5023.

[24] M. Bujko, P. Kober, M. Mikula, M. Ligaj, J. Ostrowski, J.A. Siedlecki, Expression changes of cell-cell adhesion-related genes in colorectal tumors, Oncol. Lett. 9 (6) (2015) 2463-2470.

[25] P. Clermont, L. Sun, F. Crea, K.L. Thu, A. Zhang, A. Parolia, et al., Genotranscriptomic meta-analysis of the Polycomb gene CBX2 in human cancers: initial evidence of an oncogenic role, Br. J. Cancer 111 (2014) 1663-1672.

[26] J. De Melo, V. Wu, L. He, J. Yan, D. Tang, SIPL1 enhances the proliferation, attachment, and migration of $\mathrm{CHO}$ cells by inhibiting PTEN function, Int. J. Mol. Med. 34 (3) (2014) 835-841.

[27] Y. Zhang, H. Huang, H. Zhou, T. Du, L. Zeng, Y. Cao, et al., Activation of nuclear factor $\kappa \mathrm{B}$ pathway and downstream targets survivin and livin by SHARPIN contributes to the progression and metastasis of prostate cancer, Cancer 120 (20) (2014) 3208-3218.

[28] L. He, A. Ingram, A.P. Rybak, D. Tang, Shank-interacting protein-like 1 promotes tumorigenesis via PTEN inhibition in human tumor cells, J. Clin. Invest. 120 (6) (2010) 2094-2108

[29] L. Chang, P.H. Graham, J. Ni, J. Hao, J. Bucci, PJ. Cozzi, et al., Targeting PI3K/Akt mTOR signaling pathway in the treatment of prostate cancer radioresistance, Crit. Rev. Oncol. Hematol. 96 (3) (2015) 507-517.

[30] S. Mabuchi, H. Kuroda, R. Takahashi, T. Sasano, The PI3K/AKT/mTOR pathway as a therapeutic target in ovarian cancer, Gynecol. Oncol. 137 (1) (2015) $173-179$.

[31] W. Lim, S. Park, F.W. Bazer, G. Song, Apigenin reduces survival of choriocarcinoma cells by inducing apoptosis via the PI3K/AKT and ERK1/2 MAPK pathways, J. Cell. Physiol. 231 (12) (2016) 2690-2699.

[32] O. Balat, I. Kutlar, A. Ozkur, K. Bakir, F. Aksoy, M.G. Ugur, Primary pure ovarian choriocarcinoma mimicking ectopic pregnancy: a report of fulminant progression, Tumori 90 (1) (2004) 136-138.

[33] L.Z. Jiao, Y. Xiang, F.Z. Feng, X.R. Wan, J. Zhao, Q.C. Cui, et al., Clinical analysis of 21 cases of nongestational ovarian choriocarcinoma, Int. J. Gynecol. Cancer 20 (2) (2010) 299-302 